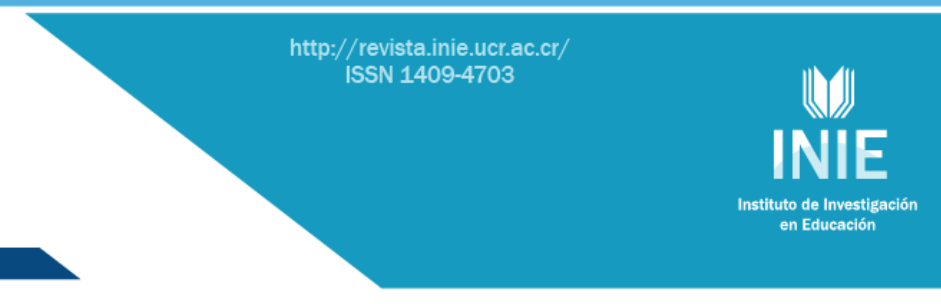

\title{
LA VIDEOCONFERENCIA DE ESCRITORIO COMO UNA HERRAMIENTA PARA EL DESARROLLO Y COLABORACIÓN A DISTANCIA \\ DESKTOP VIDEOCONFERENCING AS A DEVELOPMENT AND REMOTE COLLABORATION TOOL
}

\author{
Volumen 14, Número 2 \\ Mayo - Agosto \\ pp. 1-21
}

Este número se publicó el 30 de mayo de 2014

Ana Aurora Vidal Martínez

F. Alfonso Aguilar Valenzuela

Revista indizada en REDALYC, $\underline{\text { SCIELO }}$

Revista distribuida en las bases de datos:

CATÁLOGO DE LATINDEX, IRESIE, CLASE, DIALNET, DOAJ, E-REVIST@S, SHERPA/ROMEO, QUALIS, MIAR

Revista registrada en los directorios:

ULRICH'S, $\underline{\text { REDIE, RINACE}, ~ O E I, ~ M A E S T R O T E C A, ~ P R E A L, ~ C L A S C O ~}$ 


\title{
LA VIDEOCONFERENCIA DE ESCRITORIO COMO UNA HERRAMIENTA PARA EL DESARROLLO Y COLABORACIÓN A DISTANCIA \\ DESKTOP VIDEOCONFERENCING AS A DEVELOPMENT AND REMOTE COLLABORATION TOOL
}

\author{
Ana Aurora Vidal Martínez $z^{1}$ \\ F. Alfonso Aguilar Valenzuela ${ }^{2}$
}

\begin{abstract}
Resumen. Este ensayo propone una herramienta de interacción entre docentes y estudiantes de la Coordinación de Programas Académicos (CPA) del Centro de Investigación en Alimentación y Desarrollo, A.C. (CIAD, A.C.), situados en diferentes lugares geográficos de México, para compartir ideas, conocimiento, resolver problemas y planear estrategias de investigación. El proyecto se dividió en dos etapas. En la primera etapa se entrevistó a la coordinadora de la CPA, para conocer las necesidades específicas de interacción entre docentes y estudiantes, con el propósito de establecer las características mínimas que debería incluir la herramienta propuesta. Para el análisis de las diferentes alternativas se consideraron varios aspectos: la experiencia de los autores en el uso de equipos de videoconferencia de salón, consultas a proveedores, opiniones de colegas expertos en el área de videoconferencia de otras instituciones educativas y en bibliografía relacionada con el tema. Los involucrados coincidieron que la videoconferencia de escritorio es la herramienta que más se ajusta a los requerimientos expuestos, por considerarla: económica, ubicua y de uso simplificado para la transmisión-recepción de video, voz y datos. En la segunda etapa, después de utilizar la herramienta por un semestre, se realizaron entrevistas y encuestas a los usuarios, con el fin de evaluar el grado de satisfacción, comportamiento de docentes y estudiantes y la calidad de los materiales utilizados durante las sesiones de videoconferencia de escritorio. Los resultados obtenidos en la evaluación permiten concluir que la videoconferencia de escritorio fue la herramienta con la cual se logró alcanzar el objetivo planteado.
\end{abstract}

\section{Palabras clave: EDUCACIÓN A DISTANCIA, VIDEOCONFERENCIA, TECNOLOGÍA EDUCATIVA, MÉXICO}

Abstract. This essay proposes an interaction tool between teachers and students of the Coordination of Academic Programs (CPA) Center for Food Research and Development, AC (CIAD, AC), located in different geographical locations, to share ideas, knowledge, solve problems and plan research strategies. The project was divided into two stages. In the first stage the CPA coordinator was interviewed, for the purpose of knowing the specific needs of interaction between teachers and students, in order to establish the minimum requirements that the proposed tool should include. For the different alternatives analysis, several aspects were considered: the author's experience in salon videoconferencing, supplier's suggestions and proposals, the opinions of colleagues from other educational institutions expert in videoconferencing area and the specialized journals review. The stakeholders agreed that desktop videoconferencing is the tool that best fits the requirements exposed, considering it a budget, ubiquitous and simplified usage tool for the video, voice and data transmission and reception. In the second stage, after using the tool for a semester, interviews and user surveys were conducted in order to assess the satisfaction degree, teachers and student's behavior and the material's quality used during desktop videoconferencing sessions. The evaluation results support the conclusion that desktop videoconferencing is the right tool to achieve the stated objective.

Keywords: DISTANCE LEARNING, VIDEOCONFERENCING, EDUCATIONAL TECHNOLOGY, MEXICO.

\footnotetext{
${ }^{1}$ Actualmente labora en el Centro de Investigación en Alimentación y Desarrollo, A.C. en México, como responsable del área de tecnología educativa. Maestría Formación en Docencia e Investigación para la Educación Superior, en la Universidad Nacional de Educación a Distancia de Madrid. Dirección electrónica: e-mail avidal@ciad.mx

2 Labora en Centro de Investigación en Alimentación y Desarrollo, A.C., Planeación en Tecnologías de Información y Comunicaciones, México Ingeniero Industrial en Electrónica, Maestro en Administración de Sistemas de Información, en el Instituto Tecnológico de Monterrey, Dirección electrónica: aaguilar@ciad.mx
}

Ensayo recibido: 11 de setiembre, 2013

Devuelto para corrección: 13 de diciembre, 2013

Aprobado: 15 de mayo, 2014 


\section{Introducción}

El presente ensayo recoge una experiencia de la utilización de videoconferencia de escritorio para la interacción de docentes y estudiantes en la Coordinación de Programas Académicos (CPA) del Centro de Investigación en Alimentación y Desarrollo A.C. (CIAD). EI CIAD es un centro de investigación miembro de los centros de investigación CONACYT (Consejo Nacional de Ciencia y Tecnología). El CIAD cuenta con unidades de investigación en 7 diferentes ciudades del noroeste de México, entre las que se tiene una constante comunicación entre investigadores, docentes y estudiantes.

De hecho, muchos de esos docentes e y estudiantes con frecuencia viajan a otras instituciones de investigación en diferentes partes del mundo. Por tal motivo, esta separación geográfica y movilidad requiere de mecanismos de comunicación eficientes y flexibles que permitan la interacción y comunicación continua, en un sistema en tiempo real. Dicha comunicación se realizaba a través de la videoconferencia de sala, con equipo y personal especializado, que necesitar condiciones específicas para su funcionamiento, así como de disponibilidad tanto de equipo como de personal capacitado. Este tipo de servicio, como lo comenta Watson (2001), tiene algunas características adversas como: su costo elevado y poca movilidad, de lo cual surge la necesidad de encontrar otra alternativa para la comunicación entre estudiantes y docentes de la CPA.

En consecuencia, se procedió a investigar sobre las posibles opciones, considerando que, en la actualidad, la productividad y las relaciones globales son indispensables, y la necesidad de ser eficaces y eficientes en el manejo de los recursos, tanto económicos como de tiempo, son elementos fundamentales.

Es más, de acuerdo con Lozano y Burgos (2007), respecto al uso de las nuevas Tecnologías de la Información y de la Comunicación (TIC), la educación tiene amplias posibilidades de ser esparcida a personas geográficamente dispersas y a costos bajos a través de entornos virtuales que permitan promover habilidades y conocimientos necesarios para los nuevos retos.

Por lo anterior, el objetivo general de este proyecto consistió en proponer una herramienta pertinente y efectiva para comunicar a docentes y a estudiantes de la CPA separados geográficamente, con el propósito de que el trabajo académico y de investigación no se vean interrumpidos por la movilidad de los involucrados. Las características que se consideraron para hacer el análisis de las diferentes alternativas fueron: el fácil acceso y uso, 
bajo costo, ubicua, etc., que Watson (2001) señala como razones de peso para decidirse al entrenamiento basado en Web.

De igual manera, los objetivos específicos de este proyecto fueron: el objetivo de conocimiento, de comprensión, de aplicación, de análisis, de síntesis y de evaluación. El primero comprende el conocimiento de las reglas de etiqueta para videoconferencia, manejo de lenguaje adecuado durante las sesiones y formas para aplicar la videoconferencia de escritorio en el área específica, por parte del docente. El segundo consiste en la planeación y elaboración de los materiales para la sesión de videoconferencia. En el tercero se busca que el docente desarrolle actitudes de empatía, tolerancia, respeto y responsabilidad. El cuarto busca el desarrollo de una guía de la dinámica a seguir durante el curso. El de síntesis consiste en desarrollar un esquema de capacitación para que el docente adquiera las competencias necesarias en modalidades a distancia y, por último, el objetivo de evaluación hace referencia a la aceptación por parte de los estudiantes y docentes de la modalidad de enseñanza a distancia.

\section{Antecedentes}

La nueva Sociedad de la Información, también llamada Sociedad del Conocimiento, asentada en el principio de la globalización cultural y económica y en los constantes avances científicos-tecnológicos, está presionando con fuerza en todos los estamentos universitarios, conjuntamente con las particularidades específicas de su contexto más próximo. El resultado es una evolución cada vez más acelerada de la institución para adaptarse al cambiante entorno social, que supone un replanteamiento de su propia razón de ser, de sus objetivos y servicios de los sistemas de organización, de los métodos e instrumentos de trabajo, de los planes de estudios, de la investigación que se realiza, de las competencias que debe tener su personal (Marqués, 2001).

La cultura de la actualidad es básicamente audiovisual, entonces, se habla de "una educación de imagen" propiciada por los medios masivos y de una "alfabetización audiovisual", como complemento de la alfabetización tradicional (Castillo y Cabrerizo, 2006a). Al respecto, Clares (2000) destaca que "estamos asistiendo a un profundo cambio tecnológico, y el mundo de la educación debe unirse a él por dos motivos principales: 1) por usar los mismos medios que la sociedad está usando y 2) por introducir a los educandos en ese mundo como parte de su responsabilidad". 
La educación a distancia no es una modalidad nueva, ni mucho menos, lo que ocurre es que está experimentando un notable auge debido al desarrollo de las nuevas tecnologías, sobre todo, las que se refieren a la telemática, sin duda enriquecida con el desarrollo de los sistemas multimedia que hace más atractiva y cercana la educación (Clares, 2000).

Sánchez-Arroyo (2001), señala que la videoconferencia, supone una cuarta generación en la evolución de la educación a distancia y el desarrollo de sistemas mixtos, que contemplan ambas modalidades educativas (presencial y a distancia), ya que hace posible que los participantes distantes compartan un contexto visual, mediático y en tiempo real, próximo a una situación comunicativa cara a cara.

Una videoconferencia de escritorio nos permite establecer una comunicación sincrónica, recibiendo y enviando señales de audio y video de una computadora a otra, mediante la utilización de un software, o a través de algún servicio web.

El video o la imagen es mostrada por las aplicaciones que se encuentran ejecutándose en la otra computadora y los datos son intercambiados a través de la pizarra. Granda (2008) menciona que los sistemas más avanzados habilitan a más de dos personas para trabajar concurrentemente en un espacio compartido, incluyendo algunas características como mecanismos de transferencia de archivos, una pizarra compartida o una ventana de chat.

Se considera que la videoconferencia de escritorio puede ser aplicada en las siguientes situaciones educativas: realización de tutorías, seminarios, facilitar el trabajo colaborativo entre el alumnado, facilitar el trabajo colaborativo con otras instituciones, clases, exámenes, etc.

Además, algunos de los beneficios derivados de la utilización de la videoconferencia de escritorio son ahorro en tiempo, mejora el proceso de toma de decisiones, acelera la resolución de problemas o asuntos, permite reunir personal de lugares lejanos, reduce el desgaste humano, reduce gastos, facilita la transmisión de información, permite grabación de las sesiones para su revisión o visualización posterior, ofrece un control directo de las herramientas disponibles, sea una presentación, una demostración, un documento, etc., logra una mejor interacción entre los usuarios ante la posibilidad de utilizar video. De los anteriores, Ramírez (2008) destaca el ahorro de tiempo y dinero y la comunicación directa con las personas. 


\section{Aspectos básicos de la videoconferencia de escritorio}

El propósito de este apartado es introducir al lector en los aspectos técnicos básicos necesarios para el desarrollo e implementación del proyecto videoconferencia de escritorio en la CPA, e identificar su potencial comunicador, los beneficios que se obtienen con una herramienta de esta naturaleza y las limitaciones propias de las tecnologías que conforman el proyecto.

\subsection{Requerimientos básicos para el uso de la videoconferencia de escritorio}

Para poder participar en una sesión de videoconferencia de escritorio es indispensable contar con los siguientes elementos básicos: computadora con al menos las siguientes características, procesador Pentium III, 2 GB de memoria RAM, acceso a la Internet, mínimo de $512 \mathrm{~KB}$, contar con un navegador de la Internet que tenga instalado JavaScript y ActiveX con la opción de aceptar cookies, diadema (audífonos y micrófono) o bocinas y micrófono (si se desea interaccionar con voz), cámara web (si se desea participar con video).

Si cuenta con un dispositivo de seguridad de red (Firewall) verificar, con el encargado de redes, que no tenga bloqueado el acceso a la página a la que se tiene que conectar para la reunión, debido a que es necesario instalar un software en su equipo (software muy pequeño y con licencia abierta). En el equipo es necesario tener los privilegios de administrador, es decir, en ciertas instituciones a los usuarios no se les permite instalar software en los equipos, entonces, en estos casos será necesario hablar con el administrador de los equipos para solicitar el permiso de instalación.

\subsection{Consideraciones importantes}

Es necesario considerar esta herramienta como lo que es: un medio de difusión de los contenidos de aprendizaje, los cuales solamente están envueltos en una presentación diferente, en un lenguaje audiovisual, que es amigable en su utilización, y considerar que estos instrumentos tienen una evolución continua, gracias al desarrollo tecnológico, tal y como lo comentan Llano, Ainciburu y Lázaro (2011).

Por su parte, Scott, Quick y Castañeda (2008) concluyen en la importancia de asumir el compromiso y la responsabilidad sobre el aprendizaje y la construcción del conocimiento de los usuarios de esta herramienta. La videoconferencia de escritorio es una herramienta comunicacional y educativa que puede allanar algunas dificultades del proceso de enseñanza 
y aprendizaje, siempre partiendo de las necesidades detectadas en los planes de estudio, pero, sobre todo, de las necesidades sentidas de quien aprende, o sea, los estudiantes.

\subsection{Beneficios y limitaciones}

La videoconferencia de escritorio no debe tomarse como un reto tecnológico, sino como una nueva forma de comunicación. Salinas (2012), reconoce a la videoconferencia de escritorio como uno de los sistemas que ofrece claras ventajas para su explotación en experiencias de formación, entre las que se pueden mencionar: la facilidad para realizar tutorías, seminarios, clases, exámenes, trabajo colaborativo entre estudiantes, con otras instituciones, ahorros en tiempo, mejora el proceso de toma de decisiones, acelera la resolución de problemas o asuntos, reúne personal de lugares lejanos, reduce el desgaste humano, reduce gastos, facilita la transmisión de información, permite la grabación de las sesiones para su revisión o visualización posterior.

La productividad y el ahorro también pueden ser vistos como hacer más y mejor con los mismos recursos, en lugar de hacer lo mismo con menos.

Sin embargo, la videoconferencia de escritorio al igual que otras herramientas tiene sus limitaciones, ya que la calidad de la comunicación depende del ancho de banda del enlace utilizado tanto por los estudiantes como por el docente, la saturación que tenga la red, así como las características físicas de los equipos en ambas partes, tales como el microprocesador, memoria RAM, tarjeta de video, configuración del equipo y de sus periféricos.

\section{Desarrollo del proyecto}

El desarrollo de este proyecto se llevó a cabo en dos etapas. En la primera etapa se establecen las características y los objetivos del servicio requerido, se hace un análisis de la oferta tecnológica disponible para seleccionar la opción que cumpla con las necesidades, y se aplica la Taxonomía de objetivos cognoscitivos de Bloom, que Castillo y Cabrerizo (2006b) la consideran como una de las taxonomías más conocida y divulgada para este tipo de estudios; asimismo, se llevó a cabo el diseño y desarrollo del proyecto de videoconferencia de escritorio. La segunda etapa evalúa el impacto de la herramienta seleccionada en la etapa anterior, utilizando una metodología cualitativa de investigación-acción, que fue considerada como la más adecuada para el desarrollo de este trabajo. 


\subsection{Primera Etapa}

La primera etapa inició con una entrevista a la coordinadora del programa de posgrado de CIAD, con el fin de llevar a cabo un análisis de la situación actual y definir las necesidades de interacción entre estudiantes y docentes. Como resultado de la entrevista se determinó que la CPA requería de un sistema que tuviera las mismas funciones que ofrece el servicio de videoconferencia de sala, y que, además, cumpliera con las características establecidas, tales como: facilidad de instalación, sin costo para los usuarios, con funciones de administración que permitan gestionar de manera centralizada las características y funcionalidades para cada usuario y que brinde seguridad en la transmisión/recepción de la información.

Considerando las características anteriormente expuestas, la experiencia de los autores en videoconferencia de salón, las consultas a proveedores, las opiniones y sugerencias de colegas expertos en el área de videoconferencia de otras instituciones educativas y en bibliografía relacionada con el tema, se concluyó que un sistema de videoconferencia de escritorio es la herramienta más adecuada para el logro del objetivo general propuesto.

Posteriormente, se procedió a hacer un análisis comparativo de 4 sistemas de videoconferencia de escritorio: GoTo Meeting, Microsoft Office Live Meeting, WebEx Meeting Center y FUZE Meeting, evaluando las siguientes características: seguridad de la información, calidad de audio y video, ayuda y soporte, facilidad de uso, número máximo de participantes, soporte remoto, manuales en línea, uso compartido de aplicaciones y herramientas, compatibilidad, configuración ajustable y precio. Este proceso se llevó a cabo basado en reportes y recomendaciones de empresas independientes como: la versión de 2012 del reporte comparativo de las 10 principales empresas proveedoras de software de videoconferencia a través de Web (Business-Software.Com, 2012), Business-Software.Com (2012), Webconferencing.Org (2011) y del análisis realizado por Urbina, Arrabal, Martín, Ordinas, Pons, y Rodríguez (2010).

Del análisis anterior se pudo encontrar que, en general, las distintas opciones disponibles de software de videoconferencia de escritorio cuentan con las características requeridas. Finalmente, se decidió utilizar WebEx Meeting Center de la empresa CISCO, por recomendaciones de otros usuarios, una buena experiencia en cuanto a su desempeño en la fase de pruebas y un buen nivel de soporte técnico y asesoría disponible por parte del proveedor. WebEx Meeting Center es una solución de tipo outsourcing y es una empresa ubicada en los Estados Unidos que ofrece una plataforma sólida de acceso a sus servidores y 
una página personalizada para ofrecer el servicio de videoconferencia de escritorio, a la cual pueden acceder múltiples usuarios, para llevar a cabo la sesión de videoconferencia. La administración de las videoconferencias, conexiones y permisos, las realiza el responsable de videoconferencia de la empresa que contrata el servicio. Los servicios que ofrece WebEx Meeting Center se orientan, principalmente, a dos estrategias tecnológicas que apoyan a las tecnologías e-Business: e-Meeting y e-Learning (Webex, 2011).

Una vez seleccionada la herramienta más adecuada para cumplir con las necesidades de la CPA, se desarrollaron los objetivos específicos del proyecto, basados en la Taxonomía de objetivos cognoscitivos de Bloom que a continuación se detallan:

Conocimiento: Conocer las reglas de etiqueta para una videoconferencia de escritorio, conocer el lenguaje adecuado para la videoconferencia, conocer las diferentes formas de aplicar la videoconferencia en su área específica.

Comprensión: Planear y elaborar los materiales para la videoconferencia de escritorio, de tal manera que el estudiante entienda lo que se le está comunicando.

Aplicación: Desarrollar actitudes de empatía, tolerancia, respeto y responsabilidad durante la sesión de videoconferencia, desarrollar competencias de actitud, de valores, de conceptos y de habilidades, desarrollar la enseñanza con distintos espacios y recursos, laboratorios de medios audiovisuales, redes, etc.

Análisis: Desarrollar una guía de la dinámica a seguir durante el curso, que incluya: las diferentes actividades a realizar, responsables a contactar dependiendo de la necesidad, calendarios de fechas importantes, etc.

Síntesis: Desarrollar un esquema de capacitación para que el docente adquiera las competencias necesarias en modalidades a distancia, combinar elementos de capacitación e instrucción que den soporte de manera tal que provean un aprendizaje.

Evaluación: Determinar el nivel de aceptación de esta modalidad de educación a distancia Después del establecimiento de los objetivos, se diseñó el proyecto de videoconferencia de escritorio que comprendió: la adquisición de licencias de uso del sistema, adquisición de accesorios como cámaras, tarjetas de video, micrófonos, convertidores y conectores; se instituyeron las políticas y procedimientos de uso; se evaluaron las capacidades tecnológicas y de redes del centro y su acceso a la Internet; se dio a conocer a la comunidad que el servicio estaba disponible y el mecanismo para solicitarlo; fueron desarrollados dos programas de capacitación, uno dirigido al personal docente con temas principalmente 
enfocados a desarrollo de habilidades docentes en ambientes virtuales; preparación de material para la modalidad a distancia; conocimiento amplio de las herramientas disponibles en el software de videoconferencia de escritorio. El programa diseñado para los estudiantes es una capacitación introductoria, ya que esta se da solo con unos pocos días antes de la sesión de videoconferencia, la cual contempla solo las herramientas básicas del uso del software de videoconferencia de escritorio, para ello, fue diseñada una guía rápida la cual se envía a cada usuario antes de cada sesión de videoconferencia de escritorio.

\subsection{Segunda etapa}

Para la evaluación del impacto del resultado de la tecnología educativa implementada (videoconferencia de escritorio utilizando el software WebEx), se utilizó la metodología cualitativa. Su empleo es en función del objeto de conocimiento, el cual condicionará el tipo de estudio que se requiere llevar a cabo, con el fin de alcanzar los objetivos propuestos.

Esta metodología se orienta a describir e interpretar los fenómenos sociales y educativos, interesándose por el estudio de los significados e intenciones de las acciones humanas, desde la perspectiva de los propios agentes sociales. Se sirve de las palabras, de las acciones y de los documentos orales y escritos para estudiar las situaciones sociales, tal y como son construidas por los participantes. Los datos se recogen de forma natural, preguntando, visitando, mirando, escuchando y no en el laboratorio $u$ otros lugares controlados. El investigador se sitúa en el lugar natural donde ocurre el suceso. El contacto directo con los participantes y la interacción cara a cara es un rasgo distintivo predominante en este tipo de investigación, sea cual sea el problema de estudio que se plantee (Albert, 2007).

En el procedimiento para la evaluación se utilizó el método investigación-acción, que se consideró más acorde con los objetivos de este trabajo, ya que se orienta a la mejora de la acción educativa y contribuye a la solución de la problemática (Castillo, 2008), que consiste en reunir a estudiantes y docentes separados geográficamente, con costos bajos, facilidad de uso y movilidad.

Las técnicas e instrumentos utilizados en el proceso de evaluación del servicio de videoconferencia de escritorio respondieron a una serie de características que permitieron la selección adecuada de la información requerida. En definitiva, el uso de una serie variada de 
técnicas e instrumentos resulta imprescindible si se desea llevar a cabo una evaluación sistemática, coherente y eficaz (Castillo y Cabrerizo, 2006a).

Los instrumentos utilizados para el análisis del impacto del sistema WEBEX fueron entrevistas y encuestas. Las entrevistas (Anexo 1) a los 5 docentes se basaron en un cuestionario de 20 reactivos con preguntas abiertas y cerradas agrupadas en tres áreas, su capacitación y conocimiento en esta modalidad, las habilidades del estudiante en la utilización del sistema y la evaluación del sistema de videoconferencia de escritorio como una herramienta para llevar a cabo el proceso de enseñanza y aprendizaje. Las encuestas (Anexo 2) dirigidas para los 20 estudiantes consistieron en 20 reactivos tipo cerrados donde se evaluaron 3 aspectos generales: habilidades docentes en la modalidad a distancia, material didáctico y al igual que en las entrevistas a los docentes, la evaluación del sistema de videoconferencia de escritorio como una herramienta para llevar a cabo el proceso de enseñanza y aprendizaje.

Los datos recolectados se organizaron y clasificaron en tablas, con el fin de obtener los gráficos que representan las frecuencias y porcentajes globales, permitiendo una mejor interpretación de los mismos y facilidad de análisis.

\section{Resultados}

A continuación se presentan los resultados agrupados con base en los objetivos específicos planteados en este ensayo.

Para la evaluación del objetivo de conocimiento, que involucra el conocimiento de las reglas de etiqueta, manejo de lenguaje adecuado y formas para aplicar la videoconferencia de escritorio en el área específica, por parte del docente, durante las sesiones de videoconferencia, se les pidió a los estudiantes que evaluaran el nivel de conocimientos del docente durante las sesiones de videoconferencia de escritorio.

En la Gráfica 1 se puede apreciar el comportamiento del docente durante una sesión de videoconferencia de escritorio desde el punto de vista de los estudiantes. Como se puede ver, $40 \%$ de los encuestados respondieron que el docente tuvo un comportamiento sin interferencias (CSI) durante la sesión, es decir, el docente siguió las recomendaciones en cuanto a su expresión corporal y evitó generar ruidos innecesarios, que pudieran haber afectado la transmisión. El 20\% consideró que el docente se movió mucho (SMM) y esto distrajo la atención e hizo que la imagen se distorsionara o que disminuyera su calidad. 
Ninguno de los encuestados manifestó que el docente hiciera mucho ruido (HMR), como golpear la mesa con algún objeto (pluma, los dedos, apuntador). Esto indica que los docentes se preocuparon por seguir las reglas de etiqueta, para ofrecer una mejor sesión de la videoconferencia.

Finalmente, el $40 \%$ de los estudiantes encuestados mencionaron que no se escuchó claro (NEC) durante la presentación. Esto se puede deber a varias causas, como pueden ser: que el docente se haya alejado del micrófono, problemas de la calidad de los micrófonos, mala calidad de la transmisión, intensidad baja de la voz del maestro, baja calidad de la conexión a la Internet de los estudiantes, mala calidad del equipo de audio de los estudiantes, etc., aspectos que a medida que va madurando el conocimiento por parte de docentes y estudiantes respecto a la tecnología tienden a disminuir.

\section{Gráfica 1. Comportamiento del docente durante la videoconferencia $\square \mathrm{CSI} \square \mathrm{SMM} \square \mathrm{HMR} \square \mathrm{NEC}$}

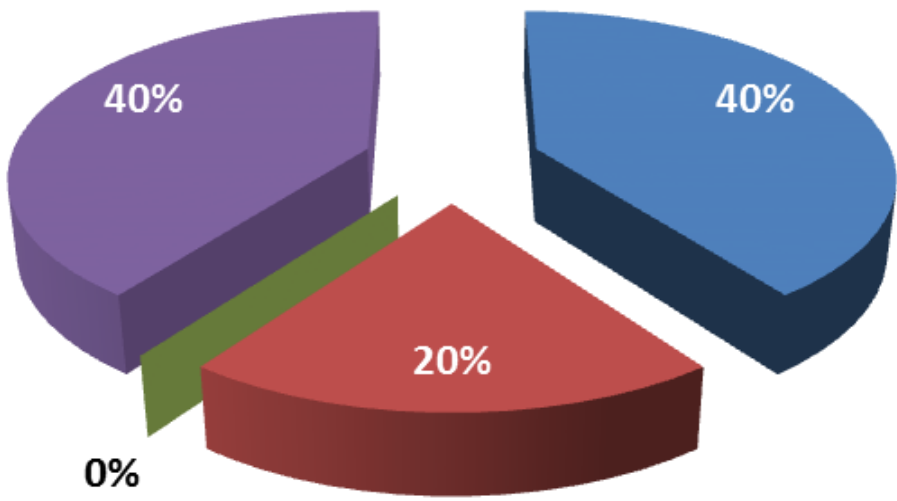

Fuente: Elaboración propia

En relación con el objetivo de comprensión, consistente en la planeación y elaboración de los materiales para la sesión de videoconferencia de escritorio destinados a facilitar que el estudiante entienda lo que se le está comunicando, se les pidió a los estudiantes encuestados que evaluaran la calidad del material utilizado por el docente, durante las sesiones de videoconferencia de escritorio, como: formato, colores, tamaño y tipos de letra, efectos, etc. Un $50 \%$ de los estudiantes calificó la calidad del material como excelente, otro $50 \%$ calificó la calidad como buena y no se recibió ninguna respuesta de regular o mala respecto a la calidad 
del material utilizado por el docente, los resultados se pueden apreciar en la gráfica 2. Esto indica que también los docentes han comprendido la importancia del material de apoyo y la calidad requerida para utilizarlos en sesiones de videoconferencia de escritorio. Estos resultados muestran la importancia de la capacitación previa a la impartición de cursos que deben recibir los docentes.

Gráfica 2. Calidad de materiales didácticos

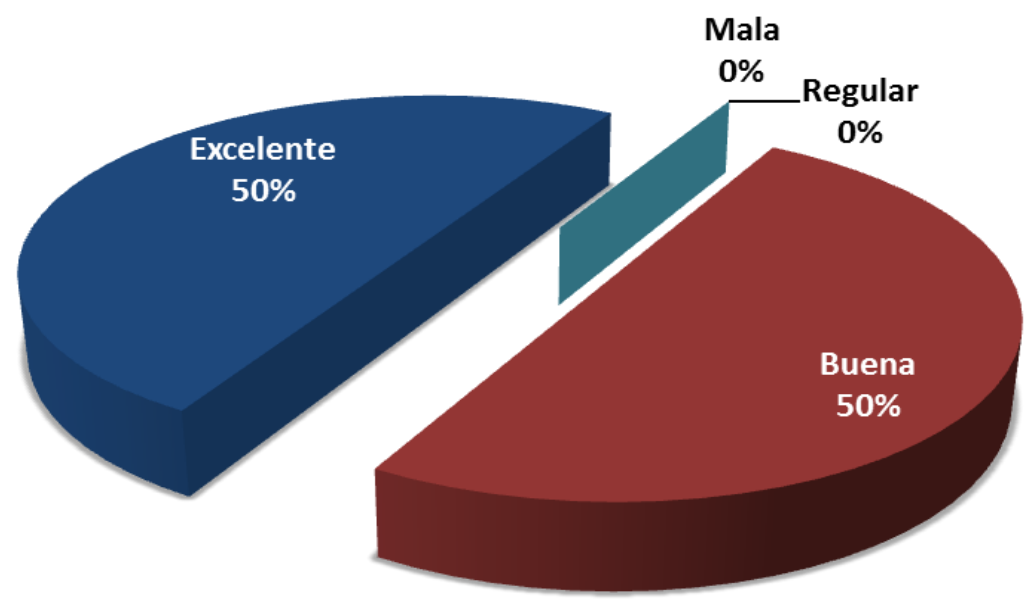

Fuente: Elaboración propia

Para que la utilización de la videoconferencia de escritorio en el proceso de enseñanzaaprendizaje sea un éxito, es necesario contar con estrategias que motiven al estudiante a participar, involucrarse y poner atención. En concordancia con el objetivo de aplicación, donde se busca desarrollar actitudes de empatía, tolerancia, respeto y responsabilidad por parte del docente, para desarrollar competencias de actitud, valores conceptos y habilidades y desarrollar la enseñanza en distintos espacios, recursos, etc., se cuestionó a los estudiantes si se sintieron motivados por el docente, durante las sesiones de videoconferencia de escritorio. El 55\% de los encuestados respondió que sí sintió una motivación por parte del docente, mientras que el $45 \%$ no sintieron ese apoyo por parte del docente.

Es importante tener presente que la videoconferencia de escritorio es, ante todo y sobre todo, un recurso, por lo que este servicio debe complementarse con estrategias didácticas, para que se lleve a cabo el proceso de enseñanza-aprendizaje. Suduc, Bizoi y Filip (2009), 
mencionan que es importante que el docente, como líder de este proceso, se esfuerce para crear una estructura que apoye el trabajo en equipo.

En el objetivo de análisis, en el cual se busca el desarrollo de una guía de la dinámica a seguir durante el curso, que debería incluir las diferentes actividades a realizar, personal con quien puede contactar el estudiante, dependiendo de la necesidad, ya sea técnica o de su materia, los calendarios con fechas importantes, etc., el 100\% de los estudiantes concordaron que este objetivo se cumplió completamente por parte del docente y la institución. Este objetivo tiene una gran importancia para facilitar que el estudiante esté enterado de la forma en que se desarrollará el curso y a quién acudir en caso de alguna duda.

El objetivo de síntesis que consiste en desarrollar un esquema de capacitación para que el docente adquiera las competencias necesarias en modalidades a distancia fue evaluado a través de varias preguntas, en las que tanto el estudiante como el docente expresaron que, aun con la capacitación recibida, es necesario continuar con un programa de cursos de actualización y metodologías didácticas para esta modalidad.

La aceptación por parte de los estudiantes y docentes de esta modalidad de enseñanza, que es parte del objetivo de evaluación, fue respondida a través de cuatro preguntas en las entrevistas y las encuestas, las cuales estaban relacionadas con: el nivel de aceptación del sistema de videoconferencia de escritorio para tomar sus cursos, si lo encontraba de utilidad como un recurso didáctico, si lo consideraron como un recurso para recibir conocimiento, información y capacitación y, finalmente, si lo recomendarían. El promedio de las respuestas fueron positivas, con un $75 \%$ de los estudiantes aceptando a la videoconferencia de escritorio como un recurso didáctico, útil y fácil de utilizar.

Dado el resultado positivo de la experiencia de utilizar la videoconferencia de escritorio como un recurso didáctico, tanto estudiantes como docentes expresaron que la recomendarían a sus colegas (Gráfica 3). 


\section{Gráfica 3. ¿Recomendaría el uso de la videoconferencia de escritorio} como una herramienta de apoyo didáctico?

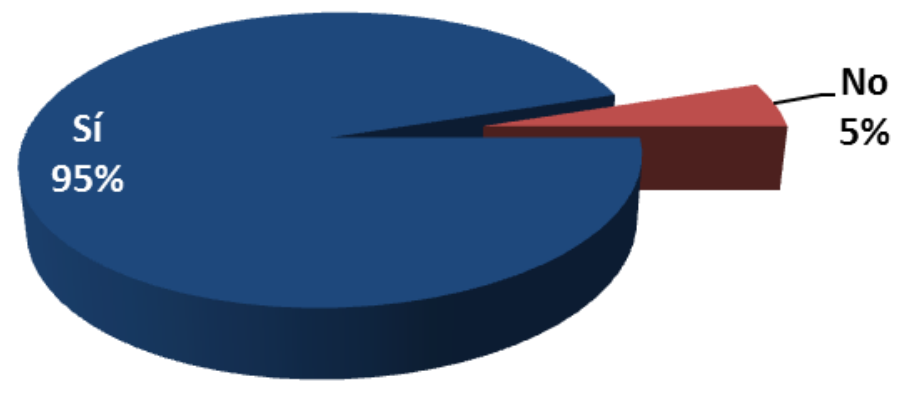

Fuente: Elaboración propia

La experiencia del uso de WebEx Meeting Center, en la CPA, brindó una serie de beneficios, aparte de aquellos dirigidos directamente al proceso de enseñanza-aprendizaje, entre los que se pueden mencionar: disminución en tiempos muertos, eliminación de gastos de desplazamiento, viáticos, hospedaje, mayor rapidez en la solución de problemas, mayor capacidad para capacitar o brindar cursos a grupos integrados por personas ubicadas en diferentes sitios, facilidad para que un docente pueda impartir su materia desde otra localidad, etc. También, la incorporación de este servicio ayudó a mantener una continuidad en la impartición de los cursos y, por lo tanto, una disminución de reprogramación de clases.

De igual manera, el uso de la videoconferencia de escritorio se traduce en ahorros al suprimir la renta de salas de videoconferencia, en los casos donde alguno de los sitios interesados en integrase a una reunión o curso no cuente con las instalaciones apropiadas.

Debido a lo económico del servicio de videoconferencia de escritorio, es posible tener varias licencias disponibles para realizar videoconferencias independientes en el mismo horario, lo que ayudó en la disminución de los casos de reprogramación de sesiones de videoconferencia, relacionados con la falta de disponibilidad de equipos y salas.

La plataforma utilizada (WebEx) ofrece la posibilidad de concertar las sesiones de videoconferencia de escritorio que se deseen, con hasta 25 participantes simultáneos o un mayor número, dependiendo del tipo de licencia adquirida.

La utilización de la tecnología de transmisión/recepción 3G y 4G garantiza una mayor cobertura y mayor calidad para la realización de las videoconferencias de escritorio, es decir, es posible realizar una videoconferencia desde cualquier sitio desde donde se pueda tener 
acceso a la Internet, que incluye los planes ofrecidos por los PSI (Proveedores de Servicios de Internet), con opciones de datos ilimitados, ofrece nuevas opciones de cobertura de los servicios de videoconferencia de escritorio.

\section{Conclusiones}

Es importante tomar en cuenta que el uso de la videoconferencia de escritorio no solo resolvió los problemas de interacción entre el docente y los estudiantes, sino que también se constituyó como una herramienta de apoyo al proceso de enseñanza y aprendizaje, la cual necesita ser respaldada por un proceso de mediación pedagógica y desarrollo de habilidades didácticas. Castillo y Polanco (2005), mencionan que con el uso adecuado de esta tecnología en los programas de educación, se puede acrecentar el papel mediador y facilitador del profesor.

El uso de esta herramienta se traduce en una disminución de costos generados principalmente por rentas de salas de videoconferencia, viajes y viáticos. Otro beneficio es el aumento de la productividad, con un mejor aprovechamiento del tiempo, ya que investigadores, académicos, estudiantes, y personal en general, están en constante comunicación. Esta tecnología es una herramienta invaluable y, desde el punto de vista educativo, representa la entrada a un mundo muy diferente, que hace que la distancia no sea un impedimento para continuar con estudios, acuerdos, evaluaciones, investigaciones con participación de varios integrantes de diferentes universidades o centros de investigación, etc.

En la CPA se ha observado que derivado de la difusión y comentarios positivos referentes al uso de la videoconferencia de escritorio, se ha generado un aumento en la demanda de este servicio para: nuevos cursos, defensas de tesis, seminarios, reuniones de comité, reuniones de trabajo, cursos de capacitación especializada al sector productivo, etc.

\section{Referencias}

Albert Gómez, María José. (2007). La Investigación Educativa: Claves Teóricas. España: McGraw-Hill / Interamericana de España, S.A.

Business-Software.com. (2012). Top 10 Web Conferencing Software Vendors 2009. Recuperado http://www.calvin.edu/ dsc8/documents/Top 10 web conferencing corporate 2009.pdf

Castillo Arredondo, Santiago. (2008). Investigación. Métodos y Recursos. Madrid, España: UNED. 
Castillo Arredondo, Santiago y Cabrerizo Diago, Jesús. (2006a). Formación del profesorado en Educación Superior: Desarrollo Curricular y Evaluación (Vol. II). Madrid, España: McGraw Hill.

Castillo Arredondo, Santiago y Cabrerizo Diago, Jesús. (2006b). Formación del profesorado en Educación Superior: Didáctica y currículum (Vol. 1). Madrid, España: McGraw-Hill.

Castillo Arredondo, Santiago y Polanco González, Luis. (2005). Enseña a estudiar... aprende a aprender. España: Pearson Educación.

Clares López Sevilla, José. (2000). Telemática, enseñanza y ambientes virtuales colaborativos. Comunicar: La comunicación Humana, 14, 191-199. Recuperado de http://issuu.com/revistacomunicar/docs/comunicar14

Granda Candás, Juan Carlos. (2008). Caracterización, Evaluación y Optimización de Sistemas Multimedia Interactivos en Entornos de e-Learning Síncrono. (Tesis de Doctorado). Universidad de Oviedo, Gijón, España. Recuperado de http://hdl.handle.net/10803/11137

Lozano Rodríguez, Armando y Burgos Aguilar, José Vladimir. (2007). Tecnología Educativa en un modelo de educación a distancia centrado en la persona. Monterrey, N.L., México: Limusa - Tecnológico de Monterrey.

Llano, Javier, Ainciburu, María Cecilia y Lázaro, Olga Juan. (2011). La Enseñanza de Español a través de Videoconferencias de Escritorio, Integración en las Diferentes Modalidades de Aprendizaje y Desarrollo de Competencias. Cuadernos Comillas, (2), 18.

Marqués Graells, Pere. (2001). Selección de Materiales Didácticos y Diseño de Intervenciones Educativas. Recuperado de http://www.peremarques.net/orienta.htm

Ramírez, Luis Alfonso. (2008). Una propuesta de plataforma para la videoconferencia de la Universidad Nacional Abierta. (Tesis Ingeniería de Sistemas), Universidad Nacional Abierta, Caracas, Venezuela. Recuperado de http://biblo.una.edu.ve/docu.7/bases/marc/texto/t35403.pdf

Salinas Ibánez, Jesús. (2012). 'I uploaded a @YouTube video’: ¿Una nueva perspectiva de la televisión educativa? Revista de Educación Mediática y TIC, 1(1), 8-28.

Sánchez-Arroyo, M. Estrella. (2001). Integración de la Videoconferencia en la Educación a Distancia. Pixel-Bit Revista de medios y educación, (17), 13 p.

Scott, Peter J., Quick, Kevin y Castañeda, Linda J. (2008). Colaboración en red a través de videoconferencia: una experiencia no formal. Pixel-Bit Revista de medios y educación, (31), 101-120.

Suduc, Ana María, Bizoi, Mihai y Filip, Florin Gheorghe. (2009). Exploring Multimedia Web Conferencing. Informatica Economica, 13(3), 5-17. 
Urbina, Santos, Arrabal, Marina, Martín, Antoni, Ordinas, Catalina, Pons, Sandra y Rodríguez, Soledad. (2010). Análisis de herramientas de videoconferencia de escritorio para la docencia en pequeño grupo y la tutoría en entornos de educación superior. Paper presented at the XIII Congreso Internacional EDUTEC 2010: E-Learning 2.0: Enseñar y Aprender en la Sociedad del Conocimiento, Bilbao, España. http://gte.uib.es/pape/gte/publicaciones/

Watson, Bradley C. (2001). The Distance Learning Project. Journal of Library Administration, 34(3-4), 325-328. doi: 10.1300/J111v34n03_12

WebConferencing.org. (2011). Web Conferencing buyer's guide- Part 1 Must have features of Web Conferencing Software. Recuperado de http://webconferencing.org/offer/webconferencing-buyers-guide-part-1-must-have-features-of-web-conferencing-software/

Webex, CISCO. (2011). CISCO Webex Administración del Sitio: Guía del usuario. In CISCO (Ed.): https://ciad.webex.com/docs/T29L/mc09011/es ES/help/ss/host/index.htm\#8753.htm 


\section{Anexo 1}

Formato para entrevista a docentes sobre el uso de la videoconferencia de escritorio.

1. ¿Aproximadamente en cuantas ocasiones ha utilizado el sistema de videoconferencia de escritorio?
a) 1-3
b) 4-6
c) $7-10$
d) más de 10

2. ¿Qué tipo de consideraciones toma para desarrollar sus materiales para los cursos en línea?

3. ¿Ha recibido algún comentario o queja de los alumnos respecto al material de apoyo utilizado durante la videoconferencia?
a) Sí
b) No Si su respuesta fue "Sí" especifique

4. ¿Toma algún criterio o consideración al momento de elegir, su vestimenta para una sesión de videoconferencia en cuanto a utilizar colores neutros, estampados mínimos y accesorio no reflejantes?
a) Sí
b) No

5. ¿Cómo realizó la estructura de su curso?

6. ¿Qué actitud adopta el alumno en una videoconferencia la mayoría de las veces?
a) Participativa
b) Pasiva
c) Insegura al uso de la tecnología

7. ¿Considera necesario que los alumnos que hacen uso del sistema de videoconferencia tomen algún tipo de capacitación?
a) Sí
b) No

8. ¿Las intervenciones de los alumnos fueron posibles en todo momento?
a) Sí
b) No

9. ¿Consideras que el alumno durante la sesión de videoconferencia tiene una actitud activa de participación?
a) Sí
b) No

10. ¿Es fácil adoptar el sistema de videoconferencia de escritorio para impartir sus clases?
a) Sí
b) No

11. ¿La ayuda que proporciona el sistema de videoconferencia ante la falta de comunicación audiovisual simultánea entre alumnos y maestros ubicados en lugares distantes es suficiente?
a) Sí
b) No Si su respuesta es "No" especifique por qué

12. ¿Recibió alguna asesoría o apoyo para impartir el curso en línea? ¿Cómo la considera? 
13. Lo aprendido por el estudiante ¿corresponde a los criterios establecidos del curso?

14. ¿Le ha gustado utilizar el sistema de videoconferencia?
a) Sí
b) No

15. ¿Le ha resultado útil el uso de este recurso para impartir conocimiento, información y capacitación?
a) Sí
b) No

16. ¿Cómo valora usted la utilidad del sistema de videoconferencia como un recurso didáctico?
a) Muy alta
b) Alta c) Media
d) Baja

17. ¿Recomendaría usted a algún colega el uso del sistema de videoconferencia?
a) Sí
b) No

18. ¿Qué problemas se le presentaron con la utilización del sistema de videoconferencia de escritorio (en cuanto a la organización de la VC, la infraestructura y el desempeño del alumno)?

19. Beneficios obtenidos al utilizar la videoconferencia (ejemplo: económicos, tiempo, menos desgaste físico, etc.).

20. Sugerencias para mejorar el proceso de enseñanza-aprendizaje a través de videoconferencia. 


\section{Anexo 2}

Encuesta aplicada a estudiantes de posgrado de la CPA de CIAD que utilizaron el servicio de videoconferencia de escritorio durante el semestre

\section{Estimado Alumno:}

La presente encuesta está formada por 20 preguntas, en donde se busca indagar aspectos de usos técnicos, didácticos y de formación de la videoconferencia de escritorio como herramienta didáctica y trabajo colaborativo en los programas de posgrado de CIAD.

1. ¿Aproximadamente en cuantas ocasiones ha utilizado el sistema de videoconferencia de escritorio?
a) 1-3
b) 4-6
c) $7-10$
d) más de 10

2. ¿Qué comportamiento adopta el maestro en una videoconferencia de escritorio la mayoría de las veces?
a) Clara sin interferencias para la transmisión
b) Se mueve mucho

c) Hace mucho ruido

d) No se escucha claro

3. ¿Qué tipo de colores en vestimenta utilizan los docentes al usar la videoconferencia de escritorio?
a) Colores neutros
b) Diseños llamativos
c) Colores cálidos

4. ¿Las presentaciones que son utilizadas por los docentes durante las videoconferencias de escritorio son legibles?
a) Mucho
b) Poco
c) Nada

5. ¿Los materiales facilitados fueron suficientes para la compresión de los temas?
a) Sí
b) No

6. ¿Los materiales utilizados durante el curso fueron suficientes para la compresión del tema?
a) Sí
b) No

7. ¿Cómo considera los conocimientos adquiridos mediante la capacitación a distancia?
a) Excelente
b) Buena
c) Regular
d)Mala e) Muy mala

8. Las evaluaciones realizadas estuvieron de acuerdo con lo aprendido en el curso?
a) Sí
b) No

9. ¿La planificación del tiempo para llevar a cabo el curso fue adecuada?
a) Sí
b) No 
10. Mi participación e intervención en las sesiones de videoconferencia de escritorio fue posible en todo momento
a) Sí
b) No

11. ¿Los docentes acostumbran dar la bienvenida a cada sitio remoto?
a) Sí
b) No

12. ¿Los docentes dan a conocer al inicio de la sesión de videoconferencia de escritorio la dinámica que se llevará a cabo, tiempo de presentación, la interacción, las actividades a realizar? (Metodología de enseñanza-aprendizaje)
a) Sí
b) No

13. ¿Consideras que el docente motiva a sus alumnos durante la sesión de videoconferencia de escritorio?
a) Sí
b) No

14. ¿Considera necesario que los docentes que hacen uso del sistema de videoconferencia de escritorio tomen algún tipo de capacitación?
a) Sí
b) No

15. ¿Es fácil aprender a utilizar el sistema de videoconferencia?
a) Sí
b) No

16. ¿La ayuda que proporciona el sistema de videoconferencia de escritorio ante la falta de comunicación audiovisual simultánea entre alumnos y maestros ubicados en lugares distantes es suficiente?
a) Sí
b) No

17. ¿Le ha gustado utilizar el sistema de videoconferencia?
a) Sí
b) No

18. ¿Le ha resultado útil el uso de este recurso para recibir conocimiento, información y capacitación?
a) Sí
b) No

19. ¿Cómo valora usted la utilidad del sistema de videoconferencia como un recurso didáctico?
a) Muy alta
b) Alta
c) Media
d) Baja

20. ¿Recomendaría usted a algún colega el uso del sistema de videoconferencia?
a) Sí
b) No 